\title{
Conduire son troupeau en silence
}

Savoirs implicites des éleveurs en production allaitante charolaise

To lead its herd in silence. The breeders' implicit know-how in Charolaise cow-

calf operation

\section{Sandrine Petit}

\section{OpenEdition}

Journals

Electronic version

URL: https://journals.openedition.org/tc/9091

DOI: 10.4000/tc. 9091

ISSN: 1952-420X

Publisher

Éditions de l'EHESS

Electronic reference

Sandrine Petit, "Conduire son troupeau en silence", Techniques \& Culture [Online], Varia, Online since 25 June 2018, connection on 29 September 2022. URL: http://journals.openedition.org/tc/9091 ; DOI: https://doi.org/10.4000/tc.9091

This text was automatically generated on 29 September 2022.

All rights reserved 


\title{
Conduire son troupeau en silence
}

\author{
Savoirs implicites des éleveurs en production allaitante charolaise \\ To lead its herd in silence. The breeders' implicit know-how in Charolaise cow- \\ calf operation
}

Sandrine Petit

Remerciements : Mes remerciements sincères vont aux éleveurs et aux professionnels d'appui qui ont accepté de me recevoir et de parler avec beaucoup de passion de leur métier alors qu'ils se sentent très incompris.

Je remercie la Chambre d'agriculture de Saône-et-Loire de m'avoir permis de participer à la formation "J'enseigne la docilité à mes bovins grâce à la méthode Souvignet ».

Enfin, je sais gré aux relecteurs pour leur lecture attentive et leurs suggestions qui m'ont permis d'enrichir le texte.

\begin{abstract}
«Quand on rentre dans une étable bien tenue, l'odeur large des bêtes est bonne à respirer, elle vous remet les idées à l'endroit, on est à sa place » Marie-Hélène Lafon 2014 Joseph, Paris : Gallimard.
\end{abstract}

\section{Un savoir implicite}

Élever et conduire des vaches seraient considérés comme allant de soi, un travail simple, tombant sous le sens commun, une «évidence invisible " (Sigaut 2012). En effet, manipuler un troupeau fait peu débat dans le conseil technique aux éleveurs ou dans le système de recherche développement. Très longtemps, la sélection génétique des animaux a ignoré les critères de comportement à cause de la lourdeur des protocoles de test (Hochereau et al. 2006). En 2015, deux critères relatifs à la docilité des animaux, basés sur l'évaluation génomique, apparaissent dans les index d'évaluation des taureaux d'insémination Charolais, c'est-à-dire bien longtemps après les indices de croissance et de productivité, et en fin de liste. Depuis janvier 2016, l'indexation en ferme des bovins allaitants (Iboval) « intègre deux nouveaux index relatifs à la docilité des reproducteurs " (Chaligne 2016). Les éleveurs sont récepteurs d'un matériel génétique performant en termes de productivité, mais qu'importe si l'animal est peu 
maniable au quotidien. Dans le conseil technique en élevage, le faire avec les animaux s'avère quasiment absent alors que l'ajustement des rations pour assurer croissance et engraissement, la santé animale, la gestion des prairies sont l'objet de prestations de services ou de formations proposées par les organismes de conseil.

Il existe toutefois quelques travaux marquants destinés à aider les éleveurs. Ainsi le manuel de Davoine et al. (2014) explique comment construire une relation de confiance avec les animaux et certaines revues professionnelles ont consacré un dossier à ce sujet (Bignon 2002). X. Boivin, éthologiste spécialiste du comportement des animaux, s'est intéressé aux élevages allaitants. Il a diffusé ses recherches sur la docilité de la vache limousine dans des revues techniques pour aider les éleveurs dans la manipulation des bêtes ${ }^{1}$. Dans les recherches en éthologie, plus développées en élevage laitier (de Passillé, Rushen 1999), on se focalise sur le comportement de l'animal et son bien-être (Boivin et al. 2012). La diversité des profils d'éleveurs et leurs savoirs s'en trouvent aplanis.

Pourtant, l'élevage est une activité exigeante et complexe, fondée sur une relation étroite avec le vivant, animal et végétal. Alimenter des troupeaux avec des ressources fourragères naturelles, faire bien pâturer, requiert des savoirs propres, des apprentissages multiples, faits d'observations, d'intuitions, de sensations, d'interactions (Dupré, Lasseur, Poccard-Chapuis 2015). Ce savoir est peu documenté. Manquant de considération dans les domaines de la recherche et du développement agricole, on peut parler d'un savoir implicite ou ignoré. L'hypothèse émise ici, est que ce savoir se transforme avec l'augmentation de la taille des cheptels et des surfaces pâturées. Une tendance que l'on observe dans les élevages de vaches allaitantes. Cet agrandissement distend le rapport de domestication de l'éleveur à chaque bête tel qu'il peut s'exprimer lors de la traite dans le cas d'un élevage laitier de petit effectif : ce qui accentue la prise de risques dans le travail (Davoine et al. 2014). En élevage extensif, quand les animaux restent une grande partie de l'année à l'extérieur, dans de grandes parcelles de prairies, l'interaction animaux-éleveur se fait plus ténue. Sur de vastes parcours boisés, les éleveurs doivent conserver le contact avec les bêtes pour qu'elles ne « s'ensauvagent " pas (Davoine et al. 2014). Ce double processus d'éloignement entre l'éleveur et ses animaux, par la taille du troupeau et le pâturage extensif, pouvant aller jusqu'à la perte de domesticité (Davoine et al. 2014), contrastent avec "l'hyperdomestication, la surprotection, la survalorisation des animaux familiers " (Digard 1990). Comment les éleveurs font-ils alors pour transférer d'un pré à l'autre des groupes de 10 à 30 bêtes, contenir et soigner un cheptel de quelques centaines de bovins d'autant que désormais ils exercent souvent seuls leur métier?

Plutôt que de laisser un fossé se creuser avec son troupeau, il semble que l'éleveur répond à cet éloignement en adaptant ses pratiques, en observant davantage le comportement des animaux. Le savoir des éleveurs se renouvelle avec l'agrandissement des troupeaux. Il présente quatre caractéristiques principales. Tout d'abord, les activités de soin et de manipulation des animaux s'avèrent dangereuses, il faut donc apprendre à éviter les risques en anticipant les réactions de l'animal. Viennent ensuite les techniques où le véritable outil de travail est le corps. L'habileté dont l'éleveur démuni d'instruments fait preuve, est celle d'amener l'animal à coopérer plutôt que de le contraindre. En troisième lieu, ce savoir est avant tout un «faire avec», une adaptation au gré des situations, un savoir-conjecturer qui invalide les connaissances 
procédurales. Enfin, et au-delà des compétences techniques, se développe une relation non dénuée d'affects, que vivent les éleveurs avec leurs animaux.

\section{Terrain et approche méthodologique}

\section{Enquête en territoire d'élevage charolais}

Cet article repose sur une enquête menée auprès d'éleveurs de vaches allaitantes, installés en Saône-et-Loire, près de Charolles et à quelques dizaines de kilomètres des agglomérations du Creusot et de Montceau-les-Mines. En 2014, le département de Saône-et-Loire comptait 222088 vaches allaitantes (Agreste Bourgogne-Franche-Comté 2016). Le nombre moyen de vaches par exploitation était de 62 en 2014 contre 57 en 2010, attestant du processus d'agrandissement des fermes. En 2010, la moyenne Unité Gros Bovins pour les exploitations de moyennes à grandes était de 131.

C'est depuis Charolles que s'étend le berceau charolais se ramifiant dans les départements voisins de la Nièvre, de l'Allier et de la Creuse. La race charolaise, réputée pour ses performances en croissance, est élevée dans l'ensemble du territoire français et à l'étranger. Jadis, la vache charolaise à la robe blanche était appréciée pour son endurance au travail des champs, sa capacité d'engraissement, sa qualité bouchère, mais aussi pour son aptitude à faire de longs trajets afin de rejoindre les lieux de vente comme Lyon et Paris (Leseigneur 1987). Au sud-ouest de Charolles, la petite région du Brionnais bénéficie d'herbages de très haute qualité, appelés embouches, riches en phosphore, enviés pour leur capacité à engraisser les bovins (Fayard 2017). En Saôneet-Loire, dès le XVIII ${ }^{\mathrm{e}}$ siècle alors que la consommation de viande augmente, les éleveurs se spécialisent soit sur l'embouche d'animaux achetés, les emboucheursmarchands, ou sur la sélection des animaux les plus performants à faire naître, les sélectionneurs, (Fayard 2017). C'est dans la seconde moitié du XX $\mathrm{XX}^{\mathrm{e}}$ siècle, que se met en place un système d'élevage dit naisseur: les éleveurs entretiennent un troupeau de vaches, dont les veaux mâles dits broutards sont vendus à l'âge d'un an, et sont pour beaucoup d'entre eux, exportés vers l'Italie où ils poursuivront leur croissance jusqu'à l'abattage. Une partie des génisses reste dans le troupeau pour assurer le renouvellement des vaches plus âgées. Ces vaches de réforme ou autres femelles peuvent être engraissées pour la boucherie, on parle alors d'élevages naisseursengraisseurs. Dans les cas étudiés, les veaux naissent à l'automne et jusqu'à mars-avril, dans des bâtiments où les vaches sont en stabulation libre ou attachées, comme c'est le cas dans les étables les plus anciennes. C'est au printemps que les veaux et leurs mères regagnent les prés pour y rester huit mois jusqu'aux premiers froids. Cette zone d'élevage est extensive. A. Leseigneur (1987), dans son étude de l'évolution de cette région entre 1955 et 1980 montre qu'elle est restée à l'écart du tournant de l'intensification productive, basée sur la révolution fourragère, la mécanisation, l'intensification de l'usage des surfaces. Le système reste extensif avec d'importantes surfaces par agriculteur et une utilisation libre de l'herbe, et donc une productivité modeste des terres et du cheptel (op.cit). Le nombre de bêtes par hectare, c'est-à-dire le chargement, reste faible : autour d'une unité gros bovin (UGB) par hectare. Plutôt que de penser à un retard dans le mouvement modernisateur de l'agriculture, ce mode d'élevage a sa cohérence et sa rationalité économique en limitant les achats externes (op.cit.). Cependant aujourd'hui, les capitaux investis dans les bâtiments, les animaux et le matériel des exploitations sont conséquents avec une rentabilité médiocre. Le prix 
des jeunes bovins vendus ou de la viande stagne, voire baisse, alors que les charges pèsent sur la comptabilité des fermes. Les revenus restent très faibles avec un résultat courant de $40 \%$ inférieur à celui de la décennie précédente (CER France 2016). Le fort endettement ${ }^{2}$ conduit à des situations dramatiques.

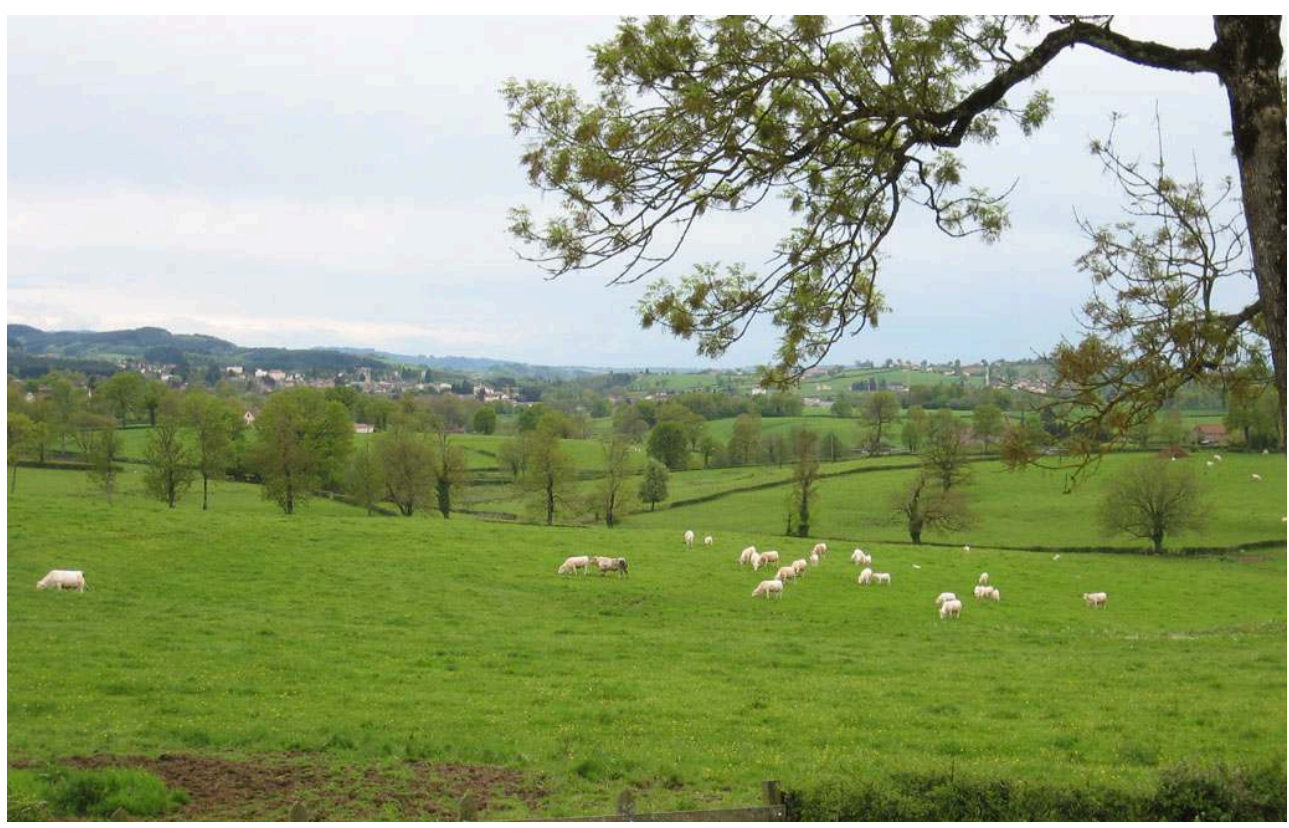

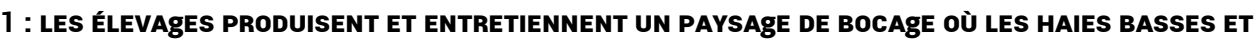
hautes délimitent des parcelles de prairies au gRÉ de Collines et de VALlons. Le paYs CHAROLAIS-BRIONNAIS EST ENgAgÉ DANS UNE CANDIDATURE DE RECONNAISSANCE AU PATRIMOINE MONDIAL DE L'UNESCO DU PAYSAgE CULTUREL DE L'ÉLEVAgE BOVIN CHAROLAIS (FAYARD, 2017 ).

(C) Sandrine Petit

\section{Méthode}

L'idée de cette enquête naît au fil de recherches menées en zone d'élevage dans le département de la Saône-et-Loire depuis 2010. Pour analyser les savoirs des éleveurs en matière de conduite de leur troupeau, j'ai adopté la méthodologie d'analyse d'un "système de pensée technique » de J.-P. Darré (1985), associant les mots et leur sens aux activités techniques. Le discours a été recueilli lors d'un entretien semi-directif d'environ deux heures auprès de cinq éleveurs et d'un inséminateur. Les entretiens enregistrés ont été intégralement retranscrits. Pour rendre compte du point de vue, je me suis attachée au « sens local» des mots (Darré 1985) (Tab. 2). Les éleveurs ont parlé de vaches, gentilles ou vives, gourmandes et curieuses; certains ont évoqué le nom d'une vache particulièrement docile comme Dom Tom, dont il a fallu se séparer, ou au contraire de Tulipe, dangereuse lors des vêlages, ou de la descendance du taureau Indien ne valant pas mieux et qu'il a fallu retrancher du cheptel. Ces termes comptent car les conduites des animaux ne sont pas seulement matérielles, mais aussi mentales ce qui suppose de «les parler, de les décrire» (Salmona 1978). Enfin, ces mots donnent accès à la manière dont les éleveurs se représentent la relation de travail avec les animaux. Relier sens et pratique a été rendu possible par l'observation participante des tâches de transport de l'étable au pré, de déplacement des génisses et des vaches d'un pré à l'autre, de tri des animaux, des soins sanitaires et d'assistance au vêlage, à l'occasion de 10 demi-journées passées à accompagner un éleveur. J'ai complété l'enquête en assistant à une formation intitulée "J'enseigne la docilité à mes bovins 
grâce à la méthode Souvignet", mise en place en août 2017 par la Chambre d'agriculture de Saône-et-Loire. Cette initiative m'a parue originale mais isolée, dans un contexte de faible prise en compte de la conduite des animaux par l'encadrement agricole. Onze éleveurs dont cinq femmes et trois techniciens étaient présents. Leurs attentes concernaient la manipulation des animaux alors que la main d'œuvre manque : «je manipule les bêtes tout seul », ou au contraire il s'agissait de trouver comment faire quand plusieurs personnes interviennent "Dès qu'on est 2 ou 3 c'est moins calme; j'aimerais les [vaches] habituer au monde ». Certains ont fait part de difficultés à approcher leurs bêtes parce que, récemment installés, ils avaient " repris un cheptel très vif», « on a repris un cheptel pas du tout facile", ou bien, pratiquant l'élevage de plein air (sans passage en bâtiment l'hiver), les bovins devenaient plus farouches. Les femmes ont déclaré que leurs conjoints manquaient de patience envers les animaux. Trois des stagiaires étaient venus pour le dressage des bêtes car, fréquentant les concours, ils souhaitaient pouvoir présenter avantageusement leurs animaux sans les laisser s'échapper. Deux éleveurs ont, par ailleurs, rapporté un accident, leurs bêtes les ayant blessés. La méthode enseignée a été mise en place par Michel et Benoît Souvignet, éleveurs sélectionneurs de vaches limousines dans le Cantal, selon leur propre expérience : "On n'a rien inventé, tout ce qu'on sait, ce sont les vaches qui nous l'ont appris et on continue d'apprendre " ${ }^{3}$. Elle est basée sur le contact avec l'animal au moment clé du sevrage des veaux où la relation à l'éleveur peut s'installer. Le second enseignement porte sur le dressage de l'animal, pour que, tenu au bout d'une corde, il puisse marcher aux côtés de son éleveur lors des concours.

\section{Des mots aux savoirs : cinq portraits}

Les éleveurs interviewés ont des profils différents (Tab.1): deux sont membres de groupements agricoles d'exploitation en commun (GAEC), familiaux, de taille moyenne, pour l'un, bien doté en main d'œuvre ; un éleveur d'une vingtaine d'années a repris seul une exploitation; enfin, les deux autres éleveurs travaillent sur des fermes de grande taille comptant 200 à 300 vaches et leur suite. Voici plus en détails, les profils des éleveurs interviewés.

5 Après une expérience d'employé dans un commerce, Jean s'est associé à l'exploitation familiale. Propriété nobiliaire, la ferme est exploitée depuis cent ans par la famille. Avec son frère, ils entretiennent quelques 100 hectares. Le troupeau est composé de 70 vaches allaitantes et de leur suite. Presqu'aucun aliment n'est acheté à l'extérieur. L'hiver, lin, céréales, pois et foin nourrissent les bêtes dont une partie est valorisée grâce au label AOC Bœuf de Charolles. Jean est fier de les nourrir des produits de la ferme. Il materne ses animaux: "nous, on va au pré, ils viennent nous lécher la culotte ». Il n'hésite pas à élever au biberon un veau et il a réservé dans ses bâtiments un espace appelé l'infirmerie qui sert à isoler les animaux blessés ou malades. La perte comme le départ des animaux sont des moments douloureux: "j'ai horreur des euthanasies, des choses comme ça »; "et puis après on verse une larme ».

6 Simon travaille avec son frère sur l'exploitation familiale depuis 1990. L'exploitation s'est agrandie autant en nombre de bêtes, aujourd'hui 115 vaches allaitantes, qu'en surface. Mais les parcelles gagnées sont éloignées du siège de la ferme, ce qui entraîne de nombreux transports d'animaux qu'il faut acheminer en bétaillère. C'est peut-être l'une des raisons pour lesquelles Simon a fait de la docilité un des critères prioritaires 
de sa sélection : «Et donc moi j'associe docilité au fait: qu'est-ce que je peux faire de ma vache tout seul ? Si j'arrive à la manipuler. Après je ne sais pas si elle est docile ou pas, mais moi j'arrive à en faire ce que je veux et ça me convient ». Ce critère est devenu un point d'interprétation de l'hérédité génétique. Simon tient des carnets et son propre registre généalogique lui permet d'interpréter le comportement des génisses et vaches: «Je remarquais des comportements (...). Et je notais, et... il y a des taureaux que je n'ai pas pris, parce que... alors ça, ça ne se chiffre pas, c'est dur à chiffrer, donc ils ne le mettaient pas sur des catalogues, mais moi, j'observais des choses".

7 Bastien est un jeune éleveur, tout juste installé hors cadre familial. Il a repris l'exploitation d'un oncle qui vient lui donner des coups de main. Bastien détient une soixantaine de vaches allaitantes. Il souhaiterait « développer la viande » c'est-à-dire engraisser davantage d'animaux en les sélectionnant sur ce critère. Tous les matins, il fait le tour de ses prés pour voir les vaches. Il est attaché à elles, quitte à prendre des décisions peu économiques comme garder une génisse non gestante parce qu'elle est gentille ou une vieille vache docile : "on a une vache gentille qu'on met à côté de la porte, elle est là, elle a déjà 12 ans, elle ressemble à une vache ce n'est pas non plus une extra mais elle est bien, quand on va au pré elle vient nous dire bonjour ... ».

8 Après avoir mis terme à une exploitation en commun, Marc est devenu salarié dans une exploitation très productive d'une centaine de vaches par équivalent temps plein (ETP). Il a dû composer avec un troupeau déjà constitué par un autre éleveur, dans une structure où le travail est rationnalisé au mieux pour assurer l'ensemble des tâches, ce qui laisse peu de temps libre à passer avec les bêtes. Pendant un an, il a cherché à connaître le comportement des vaches et en a fait changer certaines pratiques. Dès sa prise de fonction au printemps, il constate que les vaches s'enfuient quand il arrive au pré. L'hiver suivant, il instaure de nouvelles habitudes pour rendre le cheptel plus docile et maniable : «La première semaine on faisait que de les virer des prés parce qu'il faisait sec, un passait devant et les autres derrière et on les bourlinguait en voiture, ça j'y craignais et elles étaient ensauvagées. (...) Dans l'hiver j'y ai passé du temps, il a fallu qu'elles me connaissent à la voix».

9 Alain est à la tête d'un gros GAEC comptant 300 vaches allaitantes. En 2015, son fils rejoint le GAEC avec 100 vaches Aubrac qui confortent un troupeau de vaches de race charolaise. Les exploitants pratiquent la culture sur près de la moitié des terres. Une partie des récoltes sert à nourrir le bétail dont l'ensemble est vendu en tant que bêtes engraissées. L'arrivée du fils dans le GAEC introduit de nouvelles façons de procéder avec les vaches. Son père dit de lui qu'il fait ce qu'il veut des vaches et qu'il n'est d'ailleurs pas assez prudent avec elles. Il compare les races Aubrac et Charolaise : c'est la vache Aubrac qui, une fois apprivoisée, serait la plus docile. Pratiquant le pâturage tournant, il change les vaches de prés très régulièrement ce qui facilite la familiarisation. Il dira de son cheptel : «Oui, je vais quand même pas dire très docile, parce que ce serait un mensonge! Mais relativement calme et puis approchable quoi. Je vous dis, nous, on ne va pas voir nos vaches avec des lunettes d'approche!».

\begin{tabular}{|l|l|l|l|}
\hline Éleveur & Statut, structure - personnel en ETP & $\begin{array}{l}\text { Nombre de vêlages } \\
\text { par an }\end{array}$ & $\begin{array}{l}\text { Superficie exploitation en } \\
\text { hectares }\end{array}$ \\
\hline Jean & Membre GAEC1 familial - 2,3 ETP & 70 & 102 \\
\hline
\end{tabular}




\begin{tabular}{|l|l|l|l|}
\hline Simon & Membre GAEC familial - 2 ETP & 115 & 170 \\
\hline Bastien & Exploitant individuel - 1,2 ETP & 65 & 88 \\
\hline Marc & $\begin{array}{l}\text { Salarié dans exploitation } \\
\text { individuelle-2 ETP }\end{array}$ & 210 & 220 \\
\hline Alain & Membre de GAEC familial - 5 ETP & 300 & 650 \\
\hline
\end{tabular}

Tableau 1. Caractéristiques des exploitations et des éleveurs enquêtés. Groupement agricole d'exploitation en commun

10 Le tableau 2 rend compte de la manière dont les éleveurs enquêtés nomment leurs animaux et leur attribuent des qualités. Les termes utilisés pour les désigner sont relativement courants. Pourtant, certains mots ne sont apparus que chez l'un des éleveurs. Jean est le seul à employer « accouchement » pour parler des vêlages, de même que seul, Simon parle de «lignée » afin de désigner des ensembles d'animaux. D'ailleurs les mots désignant les groupes d'animaux différencient les éleveurs attentifs à une gestion par individu de ceux au regard englobant le lot ou le troupeau. On peut remarquer que Jean ne prononce jamais le mot de «lot ». Il entretient d'ailleurs, un rapport très individualisé aux bêtes, ce qui est possible compte tenu du ratio nombre d'animaux par main d'œuvre disponible. Au contraire, Marc recourt fréquemment à la terminologie collective - lot, cheptel, troupeau - ce qui semble corrélé avec la réalité pratique d'une grande exploitation où l'on gère des groupes de bêtes. Quant aux termes qualifiant le comportement des animaux, les expressions sont relativement semblables entre les éleveurs. Certaines expressions rendent compte du contact: " on les gratte ", " tu la caresses ». Chez Jean, il y a très peu de qualificatifs négatifs du bétail alors que chez Marc, qui a eu maille à partir avec un troupeau peu docile, les termes fleurissent pour désigner les vaches dures de caractère. Si les qualificatifs relatifs à la peur et à la crainte sont évoqués par Alain et Simon, ce dernier est le seul à qualifier explicitement certaines vaches de dangereuses.

\begin{tabular}{|l|l|l|l|l|}
\hline Éleveur & $\begin{array}{l}\text { Termes } \\
\text { désignant } \\
\text { animaux par } \\
\text { ordrer de } \\
\text { fréquence de } \\
\text { citation }\end{array}$ & $\begin{array}{l}\text { Qualification } \\
\text { positive des } \\
\text { vaches }\end{array}$ & $\begin{array}{l}\text { Qualification négative } \\
\text { des vaches }\end{array}$ & Trait 1 \\
\hline Jean & $\begin{array}{l}\text { Bêtes > Vaches > } \\
\text { broutards } \\
\text { petits veaux > } \\
\text { cheptel } \\
\text { génisses }\end{array}$ & $\begin{array}{l}\text { Lèche la culotte, } \\
\text { ne bouge pas, } \\
\text { viennent à côté, } \\
\text { on les gratte, } \\
\text { Docile, gentille }\end{array}$ & $\begin{array}{l}\text { Bêtes sauvages, taureau } \\
\text { méchant }\end{array}$ & $\begin{array}{l}\text { Luotidiennement. } \\
\text { «Il y a beaucoup de travail } \\
\text { manuel [pour les nourrir, les } \\
\text { soigner] » }\end{array}$ \\
\hline Bastien & $\begin{array}{l}\text { Vaches > Bêtes > } \\
\text { veau > génisses } \\
>\text { lot }\end{array}$ & $\begin{array}{l}\text { Gentilles, calmes, } \\
\text { elles viennent } \\
\text { quand on les } \\
\text { appelle à très sociables, bêtes }\end{array}$ & $\begin{array}{l}\text { Garde une vache dite } \\
\text { sympa. } \\
\text { «Je suis diplomate et on est } \\
\text { assez conservateur » }\end{array}$ \\
\hline
\end{tabular}




\begin{tabular}{|c|c|c|c|c|}
\hline Marc & $\begin{array}{l}\text { Bêtes > Veaux > } \\
\text { Vaches > Lot > } \\
\text { cheptel > } \\
\text { taureau }> \\
\text { génisse } \\
\text { troupeau }\end{array}$ & $\begin{array}{l}\text { Viens te voir, } \\
\text { docile, tu la } \\
\text { caresses, calme, } \\
\text { curieuse, } \\
\text { gourmande }\end{array}$ & $\begin{array}{l}\text { Lève les oreilles, vive, } \\
\text { carne. charogne, } \\
\text { sauvage, } \\
\text { "essauvagée ", } \\
\text { maligne, a du vice, } \\
\text { bombarde. }\end{array}$ & $\begin{array}{l}\text { Privilégie le contact de la } \\
\text { bête, la voix, et interprète } \\
\text { les rapports de } \\
\text { domination au sein d'un } \\
\text { lot. } \\
\text { "C'est au niveau du cheptel } \\
\text { comment elles sont gérées" }\end{array}$ \\
\hline
\end{tabular}

TABLEAU 2. Termes cités par les éleveurs et traits les différenciant en termes de conduite du troupeau.

${ }^{1}$ La notion de traits est empruntée à Darré (1985 : 59) qui s'en sert pour distinguer les objets et constituer des classes. Les traits cités ici vont rendre compte des variations individuelles au sein du système commun.

\section{Un élevage et des savoirs qui changent : transmission et/ou apprentissage?}

Les éleveurs constituent ainsi, au fur et à mesure, un savoir bâti sur l'observation et l'expérience, peu mis en mots. Il se transmet partiellement par le travail dans les familles (Delbos \& Jorion 1984). Les échanges entre pairs existent mais ne semblent pas déterminants dans l'acquisition des compétences d'éleveur.

Si pour quatre des éleveurs, le métier s'inscrit dans une pratique familiale, la gestion du troupeau d'aujourd'hui ne ressemble plus à celle d'antan. "Avant, mon père avait 50 hectares, 30 vaches, plus sept, huit laitières », dit Simon. Il reconnaissait toutes les vaches au premier coup d'œil, il savait leur nom et leur généalogie. Aujourd'hui, quand la mémoire fait défaut, il s'aide de numéros, de notes et de logiciels. Selon nos interlocuteurs, plusieurs éléments expliquent la dissemblance des bêtes d'aujourd'hui avec celles d'antan. La transformation des bâtiments en est l'une des raisons. Auparavant, les vaches séjournaient l'hiver dans de petites étables où elles étaient attachées. L'éleveur passait deux fois par jour nettoyer la place et apporter le foin. Dans les plus petites étables, les vaches étant face au mur supportant le râtelier, il fallait se faufiler entre les bêtes. Les contacts étaient quotidiens, et généraient des sensations comme celles qu'exprime Joseph, commis de ferme, dans la citation épigraphe. Avec l'arrivée des bâtiments en stabulation libre paillée, les animaux ne sont plus attachés. Des lots de plusieurs bêtes sont constitués et répartis entre les travées du bâtiment dans des stalles garnies de paille. Le fourrage est distribué à l'aide d'un tracteur circulant dans un couloir central. Si l'espace est moins confiné, les contacts de proximité avec les animaux s'en trouvent restreints. L'autre raison tient à l'agrandissement des fermes, aussi bien en nombre de bêtes que d'hectares. L'éleveur dispose de moins de temps par bête à l'unité, la gestion s'effectue par lots. Tandis qu'autrefois l'été, les vaches étaient conduites d'un pré à l'autre à pied, aujourd'hui les pâturages distants de quelques kilomètres du siège de la ferme sont visités en voiture. Précisons que les fermes de la région charolaise détenaient jusque dans les années 1980 quelques vaches laitières et deux ou trois chèvres, pour la fabrication de fromages dont la vente apportait un complément de revenus aux ménages (Leseigneur 1987). La spécialisation dans la production, le changement de taille des troupeaux, l'aménagement des bâtiments, l'agrandissement du territoire exploité impliquent une forme d'éloignement éleveur-animal et, de manière concomitante, induisent de 
"nouvelles pratiques d'observation actives" "alors qu'autrefois, l'observation se faisait de soi, sans chercher" (Salmona 1978). Les éleveurs tentent de s'adapter, oublient les références antérieures, inventent des conduites d'observation et d'autres façons de faire: «enfin on s'habitue aussi à des troupeaux vifs... peut être qu'on ne se rend plus compte... ", Simon; " quand je me suis installé, on avait 80 vaches; avec 300 vaches on n'a pas plus de problèmes de comportement », Alain.

12 Le savoir d'éleveur reste en partie hérité, il provient du contact dès l'enfance avec les bêtes et du travail en famille. Ainsi Marc en a tiré des astuces: " chez mes parents on faisait tout le temps comme ça, on relâchait une vache toute seule avec un lot de laitonnes; la vache, elle a les habitudes que tu lui as transmis, tu l'appelles à la voix elle vient, tu as un seau de granulés, elle vient, tous les veaux vont la suivre et s'imprégner de cela». Cette recherche du comportement approprié par le biais du mimétisme, est tentée par d'autres éleveurs (Koehler 2012). Si Simon et Marc inscrivent leur pratique dans un savoir longitudinal (Stas \& Mougenot 2009) échafaudé au sein des familles, le savoir-faire avec les animaux n'est pourtant pas une reproduction de connaissances, car les conditions du travail ont changé. Ainsi la gestion par lot d'animaux a la primauté et permet de constituer peu à peu un savoir sur leurs comportements grégaires et leur manipulation. Pour Marc, les gros lots à partir de 20-25 vaches, "ça ne se mène pas comme un petit lot ", "ça court tout le temps»: donc il faut de bonnes conditions d'environnement (parcelles et chemins), pour pouvoir intervenir, de même qu'il faut veiller à ne pas introduire de vaches vives. C'est accepter d'apprendre avant tout de ses bêtes, tout comme celles-ci aussi apprennent de l'éleveur, et leur accorder une " autorité légitime » (Despret et Meuret, 2016). En même temps, l'approche individualisée de l'animal ne disparaît pas. Pour Alain, « les vaches qui ont un bon mufle, une gueule large, elles sont plus sympas qu'une vache qui a un long nez ». Repérer des signes de maladie sur une bête, mais aussi ses aptitudes, permet de soigner ou de trier les animaux, afin de n'en garder que les meilleurs. C'est ainsi que se forge le savoir: par des apprentissages combinant gestion collective et approche individuelle, avec un soin particulier, apporté à la composition des lots.

Entre éleveurs, le savoir-faire peut être abordé au détour d'une conservation au bout d'un pré, "c'est souvent un sujet dérivé, en disant, ah ben, je me suis embêté ce matin pour trois vaches qui n'ont pas voulu sortir, c'est une fille d'un tel [taureau], moi j'ai du mal, et toi t'as du souci? ", c'est comme cela que Simon rassemble petit à petit des informations sur le profil des taureaux et le caractère héréditaire de leur comportement. Certains éleveurs se comparent à leurs voisins. Ils citeront un collègue qui fait appel à quatre personnes pour sortir les vaches du pré ou les poursuit en quad ou en voiture jusqu'à ce qu'elles abdiquent. Même quand il y a socialisation par le dialogue entre pairs, les connaissances sont peu extériorisées. Les paroles sont contenues car le sujet est délicat : «ils [ses voisins] ont hérité d'un troupeau un peu vif, mais ils ont eu du mal à le dire, parce que ce n'est pas les choses qu'on dit ", Simon.

\section{Danger et risques dans le métier d'éleveur}

«Le taureau a été vendu comme reproducteur. C'était le 38. Il était gentil. C'est pareil, on le flattait. Là, on a six babys ${ }^{4}$. Pourtant des babys, c'est des bêtes qui bougent. On peut aller dans le pré, vous allez voir ». La proposition de cet éleveur, je l'ai d'abord décliné, sachant que ces jeunes mâles ont mauvaise réputation en termes de 
comportement. La peur m'avait empêchée d'aller au contact des bêtes. Celle-ci n'est pas inconnue des éleveurs dont le métier présente des risques.

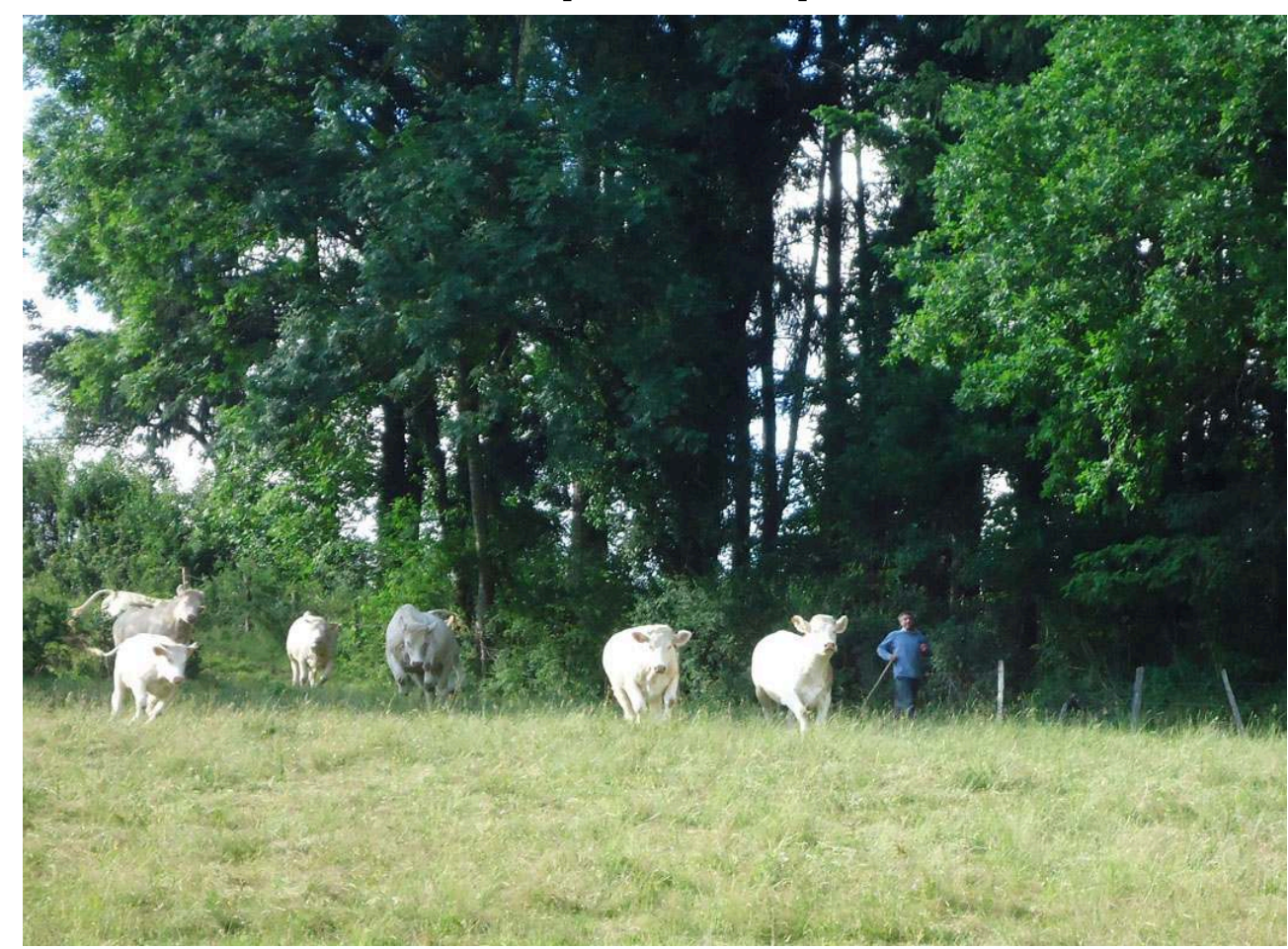

2 : En dépit de leur masse de 600 à 1000 kg, les bovins sont capables de toutes les prouesses en termes de sauts et de course.

(c) Sandrine Petit

14 La Charolaise est une vache de grande taille qui peut atteindre 1 mètre 40 à 1 mètre 50 au garrot. Les accidents avec le bétail ne sont pas rares. Selon la Mutualité Sociale Agricole (MSA 2012), parmi les chefs d'exploitation, une victime sur trois d'accidents du travail avec arrêt, exerçait une activité en rapport avec les animaux vivants et, sur les 212 chefs d'exploitation décédés par accident du travail, entre 2009 et 2011, près de la moitié étaient des éleveurs de bovins. En conséquence, la MSA promeut l'utilisation de matériel de contention, de couloir de contention et de parcs mobiles. Le sentiment de peur que les éleveurs peuvent éprouver face à leurs animaux est rarement abordé. Au contraire, les travaux d'éthologie se penchent sur celle des vaches laitières, car le stress perturbe la production (de Passillé et Rushen, 1999). Dans une enquête postale auprès de 516 éleveurs, C. Bertenshaw et P. Rowlinson (2009) examinent l'hypothèse selon laquelle le mode de relation dominant dans les fermes britanniques, est la peur que les éleveurs inspirent à leurs vaches laitières. Si l'étude montre que $72 \%$ des éleveurs pensent que les vaches n'ont pas peur des hommes, à aucun moment les auteurs n'interrogent la peur que les éleveurs pourraient avoir de leurs animaux ${ }^{5}$.

Pourtant, les éleveurs de vaches allaitantes se méfient de leurs vaches lors de la mise bas. Il s'agit d'un moment délicat où la vache essaye d'échapper à la vigilance de l'homme, alors même que celui-ci tient à l'assister.

Les vaches cherchent à se protéger du regard de l'éleveur à tel point que l'un d'entre eux prétend même que la vache, pour se soustraire à l'intervention de l'homme, se joue de l'objectif de la caméra placée dans l'étable pour une surveillance à distance. En cas de vêlage difficile, l'éleveur intervient pour tirer le veau : à la main, avec des cordes, ou 
à l'aide d'une vêleuse (un instrument qui amplifie la traction). Cette intervention nécessite parfois d'attacher la vache, et certains la placent dans un box dédié, afin de préserver sa tranquillité. Une fois le veau né, l'éleveur intervient immédiatement en l'aidant à expulser le liquide amniotique qui pourrait l'étouffer et asperge sa tête d'eau froide pour le stimuler. Puis, il laisse la vache lécher sa progéniture. Un peu plus tard, il revient pour aider le veau à prendre sa première tétée, vérifier son état de santé et désinfecter le cordon ombilical. Les veaux charolais ont la réputation d'être " mous " contrairement à ceux de race Aubrac, dont Alain dit qu'ils sont comme des petits chevreuils. J'ai pu observer l'éleveur qui approche le veau de la mamelle de sa mère, donne son propre doigt au veau pour enclencher le réflexe de succion puis glisse un trayon dans la bouche du petit. Mais cela ne se déroule pas toujours facilement, la vache tend à fuir ou à opposer sa jambe qui masque la mamelle. Parfois l'éleveur attache une vache qui bouge trop pour faciliter la première tétée, riche du colostrum. La connaissance que l'éleveur a de ses vaches, voire la confiance qu'il leur témoigne sont mises à l'épreuve à ce moment. Il faut rester en alerte : «je me suis fait charger par des vaches maternelles où je n'ai pas pu prendre le dessus, je me suis barré » (Marc). L'on m'a également rapporté le décès d'une dame, qui surveillait occasionnellement les bêtes : elle avait été mortellement chargée par une vache qui venait de mettre bas. En élevage allaitant, les aptitudes maternelles des vaches sont un critère de la sélection génétique afin que celles-ci soient des mères attentives. Très protectrices, elles défendent leur veau de toute menace et ceci peut s'avérer dangereux quand l'on s'en approche de trop près.

Beaucoup d'éleveurs, et l'intégralité de ceux qui ont été enquêtés, recourent à la fois à l'insémination artificielle et à la monte naturelle. Ils possèdent donc des taureaux pour la reproduction, lorsque les vaches sont au pré, la surveillance des chaleurs pour pratiquer l'insémination n'est alors plus possible. La force des taureaux est légendaire. Selon Simon, le taureau "ça ne pardonne pas ; ça n'a peur ni d'un bâton, ni d'un bonhomme, ni de gueuler, il te ramène à la barrière ». Il dit avoir perdu confiance avec l'un d'eux et l'a donc vendu: "une vache, on sait qu'à un moment donné, on va reprendre le dessus. Le taureau, on ne reprend rien du tout». La force des bêtes reste disproportionnée par rapport à celle d'un homme : "une bête ensauvagée de 600 kilos elle te bombarde, t'as pas le rapport de force ", Marc. Selon l'expérience des formateurs Souvignet père et fils, 10\% des animaux sont trop difficiles, des «tocards» dangereux dont il faut se séparer d'autant que leur descendance héritera des mêmes réactions. Lors de la formation, Benoît Souvignet renonça à dresser l'un des jeunes bovins mâles, dont il se méfiait. Le rapport entre l'homme et l'animal est empreint de vigilance. Les rapports de domination homme-animal tanguent et s'inversent selon les situations. La relation est davantage faite de coopération, de négociation et de ruse. 


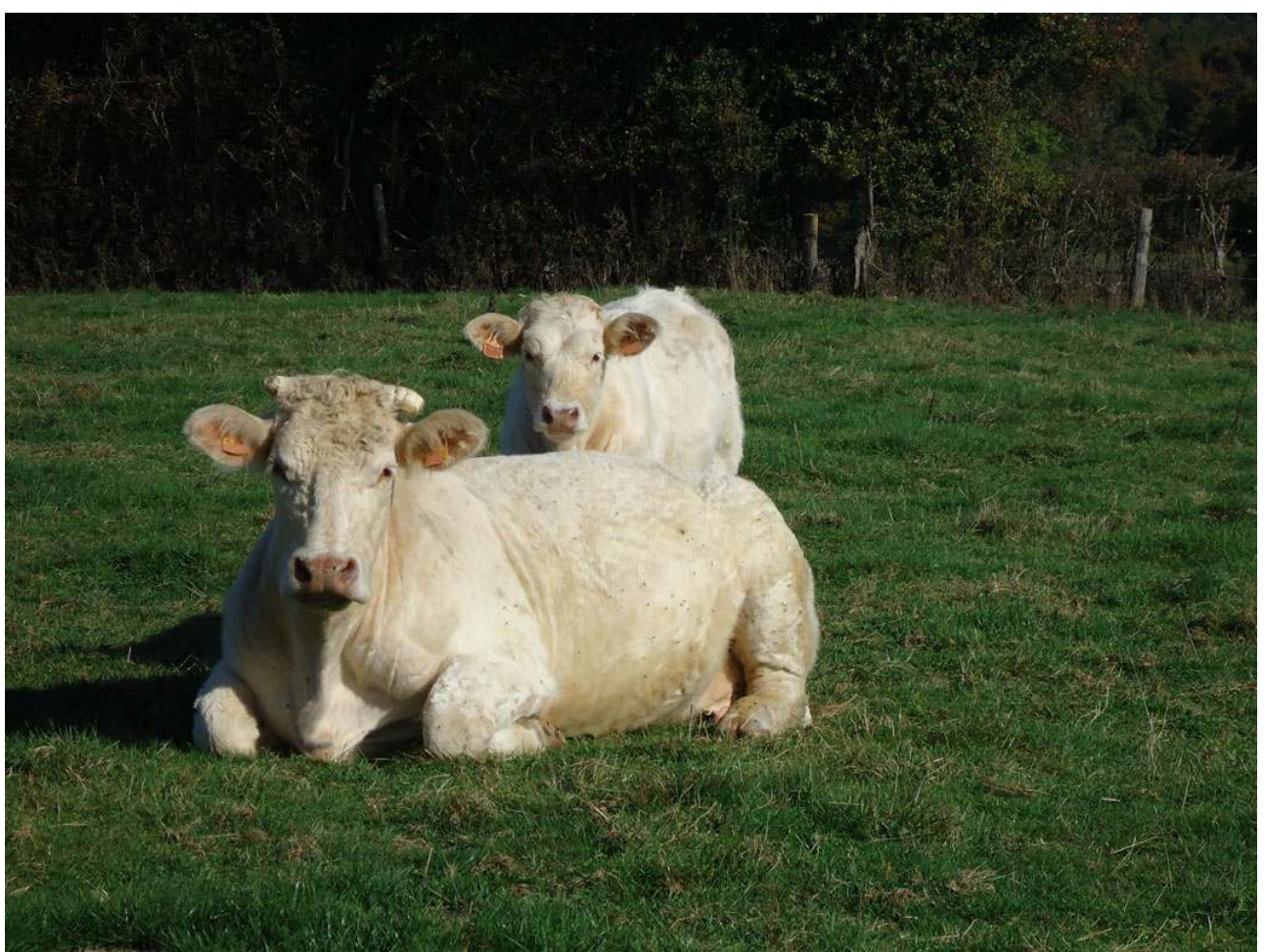

3 : Vache accompagnée de son veau. Les mères sont très protectrices et l'allaitement du veau se poursuit jusqu'à environ 8 mois, moment où il est sevré. Le sevrage est un moment clé où l'éleveur établit la relation avec les individus qui feront son troupeau. Passer au milieu des jeunes, les toucher, les attacher, leur apporter de la farine seraient autant de gages de leur docilité future.

(c) Sandrine Petit 


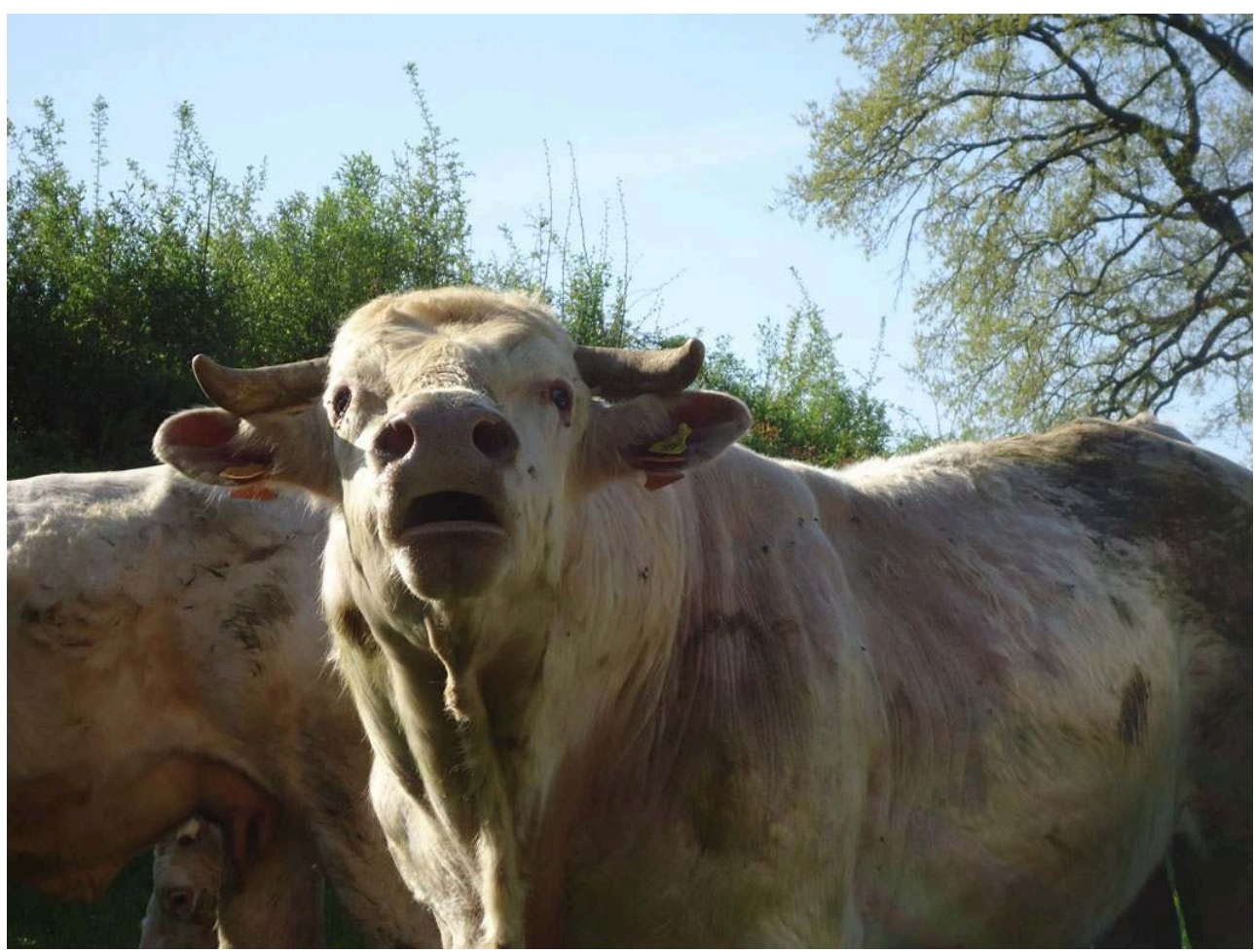

4 : Les taureaux ont tous des prénoms. Les éleveurs citent nominativement ceux qu'ils ont possédés. Ces taureaux marquent par les qualités transmises à leur descendance les lignées de vaches. Ils font la renommée des familles d'éleveurs. Leur prix varie entre 2500 à près de 10000 euros lors des ventes aux enchères.

(c) Sandrine Petit

\section{Le corps comme outil dans les actes techniques}

Pour les assister dans leurs actes techniques, les éleveurs sont peu outillés : les couloirs de contention, les véhicules de transport des animaux, les barrières cornadis et de stabulation sont les instruments du métier. Ce matériel permet d'immobiliser les animaux le temps nécessaire pour pratiquer des soins : vaccins, traitement vétérinaire ou encore pour la pesée. Le reste du temps, c'est le corps de l'éleveur qui est le principal outil (Sigaut 2012), prolongé du bâton que certains ne quittent jamais. Les tâches d'alimentation ou de déplacement des animaux, sont donc des actions « outillées » par la présence de l'éleveur à travers les actes qu'il accomplit. La main, le toucher (palper, flatter, caresser, frapper) remplit des fonctions, tout comme la voix qui hèle les vaches. Celle-ci est un repère dont j'ai pu constater l'efficacité. En mimant le « eh eh » d'appel des vaches, le troupeau a daigné me regarder tout en continuant de pâturer. Mais à la voix de leur éleveur, les vaches ont rejoint aussitôt la barrière pour changer de pré. La relation est exclusive. A. Luxereau (1995) rapporte que seul leur bouvier pouvait manier les vaches race Saint-Gironnaise des «méchantes» au caractère vif et énergique, élevées jadis en Ariège. Plus largement c'est l'attitude subtile de peur ou de confiance qui préside à la relation entre l'éleveur et ses bêtes. Ce savoirfaire avec les animaux s'ancre dans un savoir être. Chaque manière d'être et de faire est spécifique, elle fait le style de l'éleveur. L'animal en miroir réfléchit dans son comportement cette manière (Boivin, Lensink, Veissier 2000) ; tel éleveur tel troupeau, nous dira l'inséminateur. 
This media file cannot be displayed. Please refer to the online document http://

Audio : L'appel des vaches. Dans cette situation, l'éleveur appelle les vaches afin qu'elles le suivent le long d'un chemin les conduisant à une autre parcelle. II se place donc devant elles, en même temps qu'il les hèle. Les vaches ont déjà emprunté une première fois ce chemin et le suivent facilement. Les vaches se déplacent sur un chemin ombragé. C'est le printemps. Sans être perturbés par le troupeau, les oiseaux continuent de chanter.

(c) SANDRINE PETIT

19 Dans l'utilisation du corps en tant qu'outil, le regard est déterminant. Cet «œil» de l'éleveur, c'est en fait la capacité à repérer des signes rapidement. Ainsi passant devant une vache couchée, l'éleveur me dit lui trouver "mauvaise tête», car probablement malade. Pour ma part, je n'arrivais pas à voir la nuance d'expression faciale que lui avait immédiatement repéré. Ce savoir s'acquiert par une imprégnation ordinaire au contact des animaux. Mais les éleveurs diront que ce coup d'œil, cette aptitude qui fait le bon éleveur, est une qualité innée comme un don : on l'a ou on ne l'a pas.

Désormais, les éleveurs veillent à intervenir avec mesure dans les gestes et à ne pas parler trop fort. Quand «le pouvoir du travailleur avec le vivant est toujours limité ", trop faire diminuerait l'efficacité du travail (Salmona 1994). Pour transférer les bêtes d'un pré à l'autre, Simon que j'accompagne, préfère que je ne me montre pas, afin de ne pas risquer d'effrayer les vaches. En ce début d'après-midi de novembre, il s'agit de ramener un troupeau de génisses d'un pré situé à $7 \mathrm{~km}$ de la ferme. Les vaches doivent traverser une route goudronnée. Je me tiens donc à distance de la barrière, collée à la haie, en charge de stopper une voiture le temps du passage des bêtes. Les premières génisses répondent à l'appel et traversent la route sans problème, puis arrivent les retardataires. Alors que la dernière est en train de passer, par curiosité j'avance la tête et nos regards se croisent: aussitôt elle fait demi-tour et repart au fond du pré. L'éleveur la qualifie de vache vive, il me fait remarquer sa tête tenue haute et les oreilles droites. Les regards qui s'échangent comptent. Elle ne reviendra pas; il faudra attendre que les autres génisses soient reconduites dans le pré pour qu'elles finissent par traverser ensemble. Dans ce cas, l'éleveur mise sur le groupe et sur les vaches à la fois meneuses et dociles à l'appel qui entraînent le reste du troupeau. Il s'appuie sur le comportement grégaire des bêtes. Il est plus difficile d'isoler une bête d'un lot pour la conduire seule ailleurs, c'est une des raisons pour lesquelles une éleveuse était venue à la formation sur la docilité. 


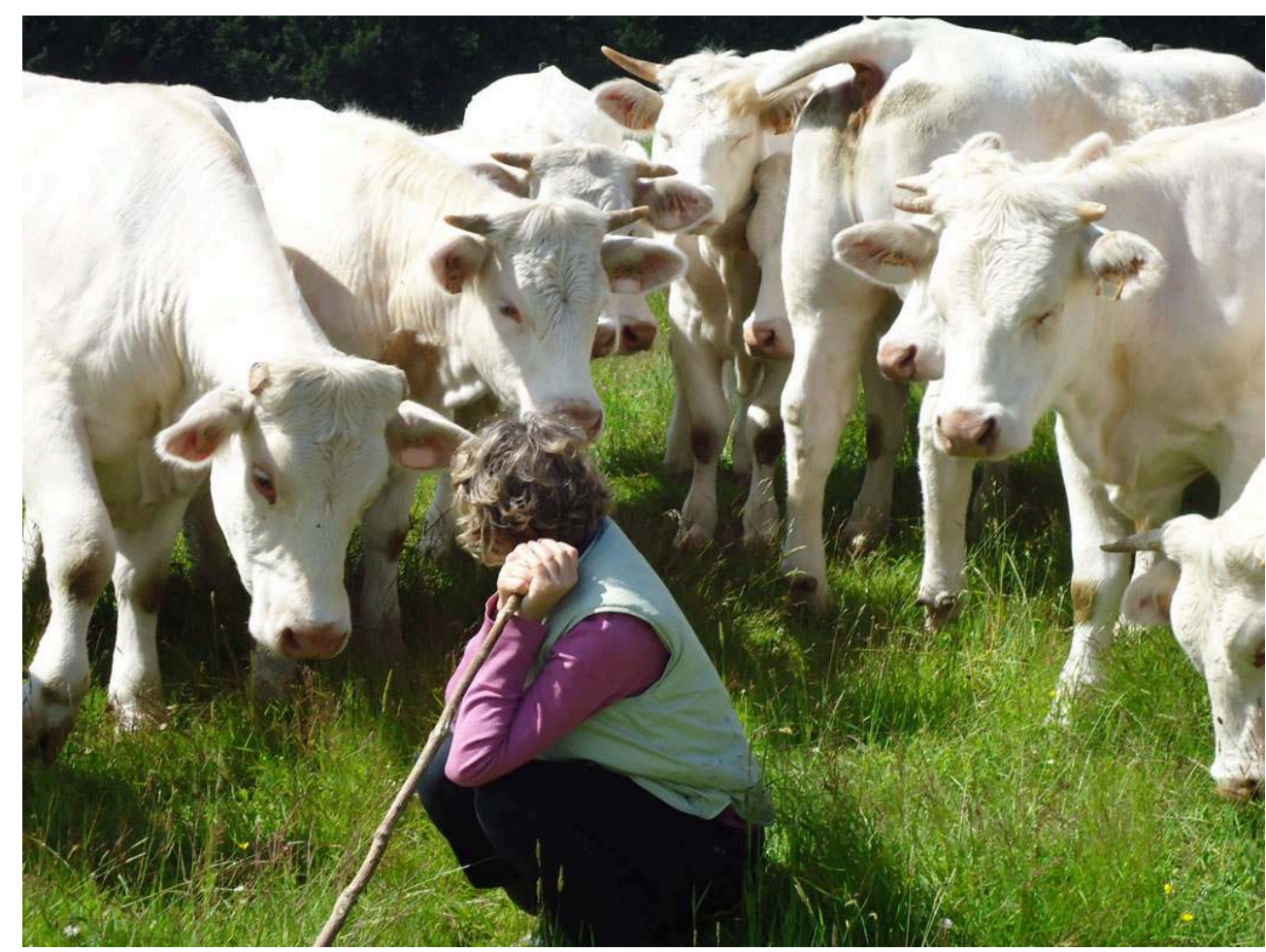

5: Visite à un lot d'une vingtaine de jeunes femelles de 1 an à 2 ans pâturant sur autant d'hectares. Les génisses sont à la fois curieuses, peureuses et gourmandes, sans être hostiles. Leur comportement résulte de la tension entre ses attitudes. Elles s'approchent pour voir. Le fait de s'accroupir permet de réduire les craintes, le moindre geste les faisant fuir en ruant.

(c) Sandrine Petit

21 Le savoir des éleveurs repose sur un faire-coopérer. Parfois, ils rusent. Ainsi, pour pouvoir conduire les vaches sur le chemin qui les ramènera à l'étable pour l'hiver, Simon rétablit la relation avec ses vaches qui s'est distendue durant l'été. Un mois avant le retour, il leur porte du foin qu'il égrène devant chacune. Elles accourent ensuite dès son arrivée. Quand vient le moment du retour, elles suivent aisément l'éleveur sur le chemin. Quand il s'agit de les faire monter dans la bétaillère, un autre tour intervient. Si possible la remorque est cachée dans la courbe du chemin, pour être tenue hors du champ de vision de l'animal. Les chemins en courbe plutôt que les lignes faciliteraient la conduite du troupeau vers un lieu de contention, une tactique que des éthologues comme Temple Grandin (2006) ont pu repérer.

Car travailler avec les bêtes c'est mettre au point des tactiques mais aussi savoir "lâcher du lest » (Salmona 1978, p.10) : «on n'insiste pas », l'expression revient dans la bouche des éleveurs. "Oui, on ne crie pas, on n'a pas de quad, de moto, si on n'y arrive pas, on ne pousse pas...Tant pis, quoi. (...) Le forçage, on n'a pas les armes pour, parce qu'on est trop loin des bâtiments, enfin, on est trop loin, on est obligés de passer par la bétaillère, on manque de main d'œuvre, parce que le forçage ça peut marcher, mais il faut être quatre, cinq, que la vache sente qu'elle est prise ", selon Simon. Il faut accepter de différer les interventions quand il vente ou que l'orage menace, ou que, pour d'autres raisons, le moment n'est pas propice. Au détour d'un chemin, Simon me montre une barrière ouverte au-delà de laquelle les vaches refusent d'aller. Sa localisation centrale ne convient pas, une position en angle serait plus juste avec une pierre de sel à lécher tout près. Le plus surprenant est que ce comportement se répète d'une année sur l'autre quelles que soient les vaches. Il faudrait déplacer la barrière pour s'adapter au comportement des 
animaux. Trouver la mesure dans la gestion du troupeau c'est aussi pratiquer le nonagir, par exemple ne pas intervenir quand les vaches se toisent ou luttent entre elles pour reconstituer la hiérarchie dans le groupe, et ce même si elles courent le risque de se blesser. Ainsi, il faut savoir « composer avec » les animaux comme l'écrivent Despret et Meuret (2016), contenir les brebis sans les forcer, c'est-à-dire «mener à faire » dans une relation complexe qui ne se résume pas à un rapport de force. Face à l'état actif des animaux, l'homme doit opter pour une attitude constante de négociation et de compromis avec eux (Digard, 1990). Cela vaut pour d'autres animaux que les bovins. Ainsi, avant le concours annuel de reproducteurs de cochons Piétrains, la « tentative de dressage " s'apparente à une négociation, les éleveurs se pliant au caractère du cochon (Stas et Mougenot 2009).

\section{Savoir-conjecturer : décrypter les signes et ajuster les gestes}

Le métier s'apprend en le voyant faire et en le faisant (Darré 1985). C'est en faisant que l'éleveur éprouve la justesse de ces gestes. Si les manuels donnent des consignes pour arriver aux meilleurs résultats, dans la pratique les éleveurs composent avec des situations inédites car le contexte n'est jamais identique. Ce savoir est un savoirconjecturer c'est-à-dire une capacité à deviner ce qui va se produire (Cornu, 1991), quand la vache ou le lot de bêtes peut réagir de manière imprévisible. Les éleveurs créent des habitudes. Elles sont le socle : « au fil du temps, elles ont appris à me connaître et moi, j'ai capté les habitudes des vaches;(...) si tu vas chercher les bêtes le matin à la même heure...c'est bien plus pratique et elles sont bien plus dociles", Marc. Mais le socle s'ébranle au moindre changement " par contre, si je change les habitudes, si on y va à deux, elles seront plus curieuses, attentives, voilà, et dès qu'on est deux ou trois, qu'on parle un peu plus fort, ... il y a quelque chose de différent, elles le sentent », dit Simon. Un «bon » marin est celui qui est le plus mobile physiquement et dans sa tête (Geistdoerfer 1999), une maxime qui vaut face au troupeau. Des habitudes, il faut décoder des messages exceptionnels et restructurer ses connaissances à chaque regard (op.cit.) car il faut prévenir l'accident quand les événements tournent à l'imprévu : «Ben j'ai dû gueuler pour les faire avancer, et puis la vache elle s'est retournée et puis... mais elle m'a chargé, chargé hein! J'étais par terre...» (Alain). L'inséminateur enquêté se rend avec appréhension dans deux exploitations sur les 140 qu'il fréquente. Sa sagacité lui permet de flairer les coups : « il faut prendre le temps de les prévenir, leur causer, elles [les vaches] savent qu'on est derrière (...) on arrive à anticiper le coup de pied, je sais qu'on arrive à les anticiper, des comportements, je ne sais pas, ça se sent, ça se voit quand elles sont agitées». La connaissance est ainsi " encorporée " (thinking through the body) (Whatmore 2002), une habileté tacite et une vigilance qui mobilisent les perceptions sensorielles. Ce savoir assimilé repose sur un mélange de tours, de coups d'œil, de dons et d'habitudes, qui rendent les ficelles du métier difficiles à transmettre.

L'art d'élever des animaux ne se résume pas à connaître leur bien-être. C'est un savoirfaire et un savoir être avec les bêtes dans des situations toujours changeantes et des relations toujours provisoires car pouvant basculer au moindre événement. Les situations changent, les bêtes changent. Ainsi les vaches en vieillissant perdent les qualités pour lesquelles les éleveurs les avaient gardées. Selon Alain, les vaches d'une dizaine d'années rusent pour ne pas rester coincées dans la barrière cornadis, ce sont 
des «vieilles malignes». Si les situations et les animaux se transforment, parfois au risque de rendre le métier dangereux, la notion de contrat de domestication tel que proposé par Larrère et Larrère (2000) s'accommode mal avec des rapports de pouvoir complexes entre l'homme et l'animal et des moments jamais identiques d'un élevage qui lui-même se transforme. Toutefois il permet d'aborder la responsabilité morale de l'éleveur de protéger et nourrir ses animaux, c'est-à-dire assurer un bien-être.

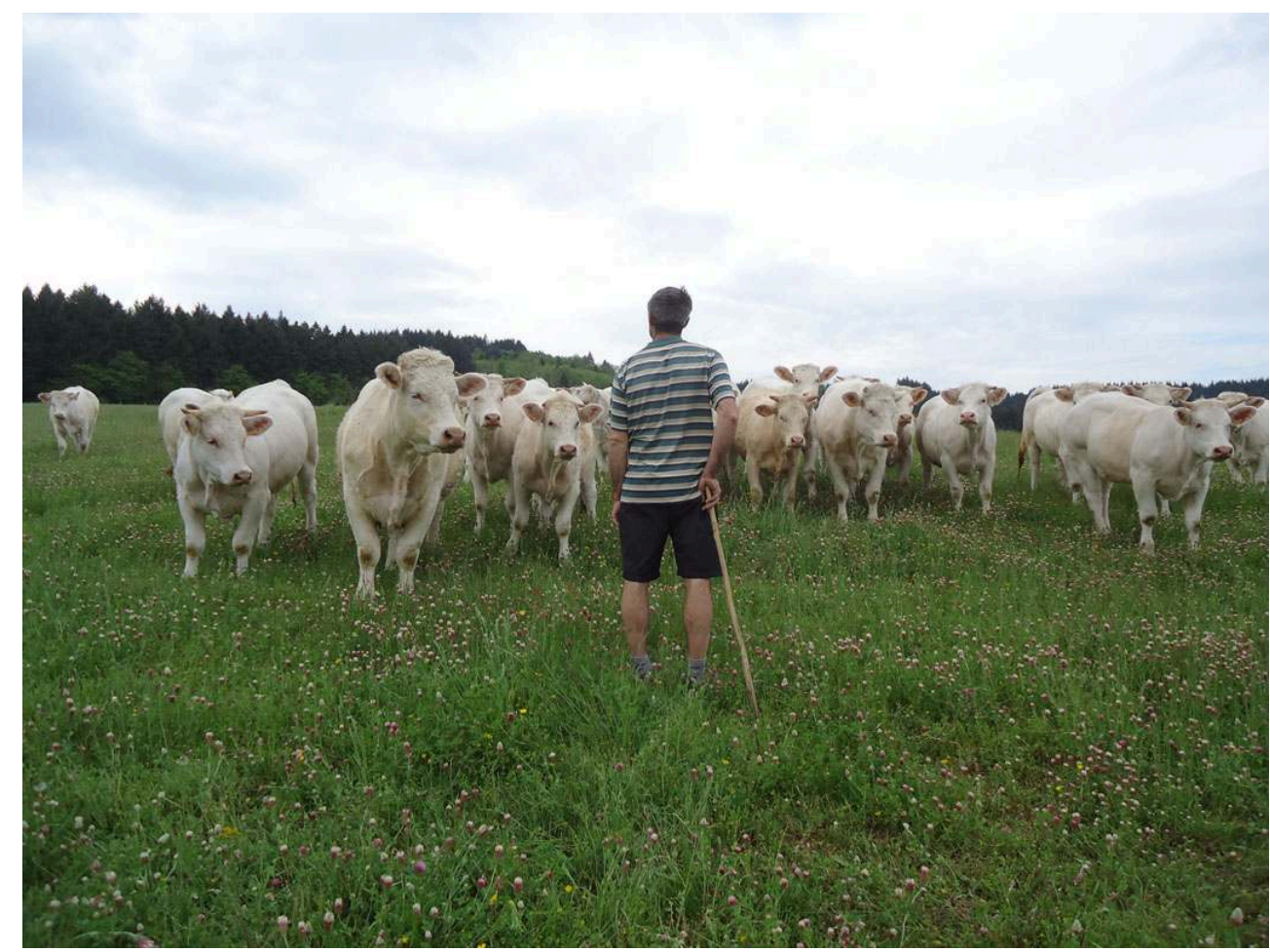

6 : L'élevage est jalonné de moments gratifiants quand les animaux coopèrent facilement ou se laissent approcher voire caresser, comme c'est le cas ici où les vaches viennent d'elles-mêmes à la rencontre de l'éleveur qui vérifie alors que toutes vont bien. Le plaisir d'Alain est de " voir les bêtes toutes couchées, elles sont d'un calme, j'adore ça ». L'élevage est aussi fait, par moments, d'échecs qu'il faut accepter quand un troupeau ou une bête refuse de changer de pré, de traverser une route. Ces deux temps font de l'élevage un travail stimulant d'autant que les vaches allaitantes sont réputées plus « sauvages » et « dynamiques » que les vaches laitières (Kohler 2012).

(c) Sandrine Petit

\section{Une dimension relationnelle forte avec le vivant}

Des revues comme Animal Welfare ou Applied Animal Behaviour Science, traitent du comportement et du bien-être animal (plutôt en production laitière pour les bovins) au travers d'une perspective où la peur ressentie par les bovins doit être circonscrite afin de permettre leur manipulation (Rushen et al. 1999), éviter les accidents et ne pas dégrader la production (Boivin \& Mounier 2009). A.-C. Dockès et F. Kling-Eveillard (2007) identifient dans le discours des éleveurs trois représentations de l'animal: l'animal instrumentalisé, le communiquant et l'affectif. Ces représentations non exclusives s'entremêlent chez les éleveurs selon les situations. S'ils aiment leurs bêtes, ils n'oublient pas de s'en méfier. S'ils aiment leurs bêtes, ils acceptent de s'en séparer. Marc au-delà de l'affect individuel redonne à son métier un sens social, produire de la viande pour le plaisir des autres : " j'y vois comme ça, tu l'as engraissé elle est ronde comme une pomme, elle est vraiment jolie (...) elle fait envie, même que je l'aime, j'ai envie de la 
manger ». Mais pour Jean, la proximité forte avec les bêtes rend leur départ douloureux "Après on en est malade quand ils sont trop gentils et qu'il faut les emmener à l'abattoir ». À l'appel, les vaches s'approchent et Simon dit d'une voix douce "allez mes belles» ou encore «allez mes cht'ites». Pour Marc, "une petite caresse, ça crée le lien ». Les éleveurs enquêtés expriment un attachement différencié pour certaines: "elle s'appelait Dom Tom, 7045 son numéro, t'arrivais dans le pré elle venait te voir et elle attendait que tu la caresses, je l'aimais pour ça mais c'était pas la meilleure des vaches en termes de production mais je l'aimais pour ça et c'te vache tu la foutais dans un troupeau, tu l'appelais, elle venait elle faisait sortir les autres bêtes, c'était une satisfaction au niveau de la relation des bêtes parce qu'on les aime parce que c'est des bêtes de rente mais aussi on aime les animaux tout simplement ", Marc. Cette personnalisation du lien à l'animal, concerne principalement les vaches qui restent plusieurs années dans le troupeau et en font son ambiance et sa réussite. Les jeunes veaux mâles destinés à quitter rapidement la ferme sont gérés en lots sans que les éleveurs cherchent à établir une relation particulière. L'approche relationnelle à l'animal n'empêche pas un pragmatisme dans l'élevage où souvent le temps manque. Plusieurs d'entre eux ont la même formule quand je leur demande ce que serait la vache idéale : "c'est une vache dont tu n'entends jamais parler (...) qui fait un veau par an, et qui l'élève, c'est ça la meilleure des vaches! ", Marc; " elle amène son veau, elle a un peu de lait, elle fait le veau toute seule...Elle revient en chaleur, en fait tu ne la vois pas, elle n'existe pas ", Simon.

\section{Conclusion : des savoirs fragiles}

Les pratiques d'élevage changent et les savoirs qui les accompagnent également. Le mouvement actuel d'agrandissement des fermes semble s'accompagner d'une inévitable distanciation entre l'éleveur et ses bêtes toujours plus nombreuses. Toutefois, les éleveurs recomposent leurs manières de faire. L'hypothèse qui se dégage de cette enquête est qu'aujourd'hui les éleveurs ont besoin de davantage de tours et de ruses pour arriver à manier des lots d'animaux pâturant sur de vastes parcelles. L'observation du comportement grégaire des animaux permet d'élaborer de nouveaux savoirs. «En produisant des animaux, on produit également de la domestication c'està-dire du pouvoir de l'homme sur l'animal » (Digard 1990). Mais dans le cadre de grands troupeaux en élevage extensif, ce pouvoir est contesté par les animaux eux-mêmes, et parfois s'inverse. Les éleveurs sont dans la recherche d'un équilibre entre un instinct naturel davantage manifesté et une proximité nécessaire. Ils apprennent à coopérer avec le vivant et non toujours à le contraindre.

Ces savoirs sont fragiles et menacés. Les moyens de contention viennent se substituer aux techniques du corps (Mauss, 1936) que l'éleveur développe au fur et à mesure dans son travail. Les disciplines comme l'éthologie, la zootechnie en privilégiant l'animal ou la production, tendent à minimiser et à effacer la place et les savoirs des éleveurs. Ceux-ci semblent cantonnés par la recherche et l'accompagnement technique agricole, au domaine des connaissances privées. Ainsi, le comment travailler avec les animaux, est-il un savoir constitué dans un contexte de faibles interactions sociales. Avouer des difficultés à ses pairs reviendrait à se délégitimer au cœur de son métier. Enfin, la situation économique actuelle laisse présager à terme, de l'abandon du métier et de la disparition des élevages. Une des voies sera-t-elle une extensification accentuée proche des pratiques de « ranching » c'est-à-dire une extensification du travail dans un espace rural aux faibles densités de population (Ingold, 1980) ? Ce scénario envisagé lors d'une 
prospective sur l'élevage allaitant de Saône-et-Loire en 2010 n'a pourtant pas séduit les éleveurs qui tiennent à continuer l'histoire des familles et de leur cheptel dans un rapport de proximité.

\section{BIBLIOGRAPHY}

Agreste Bourgogne 2012 L'élevage allaitant en Bourgogne: des troupeaux de plus en plus grands. Bourgogne : DRAAF.

Agreste Bourgogne-Franche-Comté 2016 Atlas Bourgogne-Franche-Comté. Bourgogne-FrancheComté : DRAAF.

Bertenshaw, C. \& P. Rowlinson 2009 « Exploring Stock Managers' Perceptions of the HumanAnimal Relationship on Dairy Farms and an Association with Milk Production », Anthrozoös 22, 1: 59-69.

Bignon, E. 2002 « Comportement des bovins », Réussir lait Elevage, 148 : 28-39.

Boivin, X. et al. 2012 « Hommes et animaux d'élevage au travail: vers une approche pluridisciplinaire des pratiques relationnelles », INRA Productions Animales 25, 2, 159-168.

Boivin, X. \& L. Mounier 2009 « La relation homme-animal ou comment utiliser le comportement des bovins pour les manipuler?», Le nouveau praticien vétérinaire 3, $12: 17-22$.

Boivin, X., Lensink, B.J. \& I. Veissier 2000 « The farmer and the animal: a double mirror », in M. Hovi \& M. Bouilhol eds. Proceedings of the 3rd NAHWOA Workshop. Clermont-Ferrand : University of Reading, 21-24 oct. $2000:$ 5-13.

CER France, Chambre d'agriculture de Bourgogne France-Comté, 2016 Observatoire prospectif de l'agriculture bourguignonne. Résultats 2015.

Chaligne, H. 2016 «L'indexation Iboval intègre la docilité au sevrage », La France agricole 3643 : 33. Cornu, R. 1991 " Voir et savoir », in D. Chevallier dir. Savoir et pouvoir transmettre. Paris : Éd. de la Maison des sciences de l'homme : 83-100.

Darré, J.-P. 1985 La parole et la technique. L'univers de pensée des éleveurs du Ternois. Paris : L'Harmattan.

Davoine, J.-M. et al. 2014 Domestiquer autrement. Homme-animal en élevage extensif, une relation de confiance. Cardère Editeurs.

Delbos, G. \& P. Jorion 1984 La transmission des savoirs. Paris : Editions de la Maison des Sciences de l'Homme (« Ethnologie de la France »).

De Passillé, A.M.B. \& J. Rushen 1999 « Are You a Source of Stress or Comfort for Your Cows? Advances in Dairy Technology ", 11 : 347-360.

Despret, V. \& M. Meuret 2016 Composer avec les moutons. Lorsque des brebis apprennent à leurs bergers à leur apprendre. Nîmes : Cardère éditeur (« Hors les drailles »).

Digard, J.-P. 1990 L'homme et les animaux domestiques. Anthropologie d'une passion. Paris : Fayard. 
Dockès, A.C. \& F. Kling-Eveillard 2007 « Les représentations de l'animal et du bien-être animal par les éleveurs français », INRA Productions Animales, 20, $1: 23-28$.

Dupré, L., Lasseur, J. \& R. Poccard-Chapuis 2015 « Faire pâturer, Faire société, durablement. Éleveurs et élevages, entre inscription territoriale et changements globaux », Techniques \& Culture $63: 202-231$.

Fayard, D. 2017 « L'élevage des bovins charolais. Durabilité et patrimonialisation d'un système d'élevage extensif à l'herbe dans le berceau de la race », Pour 231 :167-177.

Geistdoerfer, A. 1999. « Aimer la mer : une technique du corps et de l'esprit », in J.-L. Jamard, A. Montigny \& F.-R. Picon dir., Dans le sillage des techniques. Paris : L'Harmattan : 153-172.

Grandin, T., Johnson, C. \& I. Farny 2006 L'interprète des animaux. Paris : Odile Jacob.

Hochereau, F., Boivin, X. \& H. Brives 2006 « La docilité : un nouvel indicateur de sélection animale pour une agriculture durable? », Entreprises et Histoire $45: 113-117$.

Ingold, T. 1980 Hunters, pastoralists and ranchers. Cambridge : Cambridge University Press ( Cambridge studies in social anthropology»).

Kohler, F. 2012 « Blondes d'Aquitaine. Essai de zooanthropologie », Etudes rurales 189 : 155-174.

Larrère, C. \& R. Larrère 2000 « Animal rearing as a contract ? ", Journal of Agricultural and Environmental Ethics $12: 51-58$.

Leseigneur, A. 1987 Evolution de la petite région agricole « charolais » (1955-1980), Document de recherche Inra n³4, $96 \mathrm{p}$.

Luxereau, A. 1995 « Les vaches d'avant étaient méchantes celles de maintenant sont des bâtardes. Changement de races en Couserans (Pyrénées ariégeoises) », in B. Lizet, B. \& G. Ravis-Giordani dir. Des bêtes et des hommes. Paris : Editions du C.T.H.S. : 93-105.

Mauss, M. 1936 « Les techniques du corps », Journal de psychologie XXXII, 3-4. u

MSA 2012 Statistiques des risques professionnels des non-salariés et des chefs d'exploitation agricole. Données nationales 2011.

Rushen, J., Taylor, A. \& A.-M. de Passille 1999 « Domestic animals' fear of humans and its effect ontheir welfare », Applied Animal Behaviour Science 65: 285-303.

Salmona, M. 1978 L'homme et la vache. Paris : ITEB - CAESAR, n79.061.

Salmona, M. 1994 Les paysans français. Le travail, les métiers, la transmission des savoirs. Paris :

L'Harmattan.

Sigaut, F. 2012 Comment Homo devint faber. Paris : CNRS Editions.

Stas, S. \& C. Mougenot 2010 « Les concours de cochons « Piétrain». Regard anthropologique sur une race au carrefour de son histoire », ethnographiques.org 19. [En ligne] :

www.ethnographiques.org/2009/Stas-Mougenot1.

\section{NOTES}

1. Dans la revue Bovins Limousins ( $\mathrm{n}^{\circ} 170$ en $2007, \mathrm{n}^{\circ} 187$ en 2011).

2. http://idele.fr/no_cache/recherche/publication/idelesolr/recommends/situation-financieretoujours-difficile-pour-les-exploitations-bovines-a-lete-2017.html.

3. L'exploitant agricole de Saône-et-Loire, n² 2762, 28 juillet 2017. 
4. Jeune bovin mâle d'élevage, dont l'abattage pour la consommation a lieu vers 20 mois.

5. Dans les verbatim de réponses mentionnées dans l'article, un éleveur pourtant, fait part de vaches qui peuvent tout à coup devenir dangereuses, comme si elles n'avaient plus aucune peur.

\section{ABSTRACTS}

Dans un contexte d'agrandissement des troupeaux, l'éleveur doit renouveler sa relation aux animaux et son savoir en matière de conduite des troupeaux. Le comportement des bêtes est déterminé par les habitudes qu'il leur inculque, mais aussi par l'hérédité génétique. Le savoir de l'éleveur réside dans l'observation du comportement grégaire des bêtes qui s'exprime davantage en élevage extensif. Il développe des rapports de coopération avec ses vaches car il n'est plus possible de les contraindre. Il a aussi ses tours. Ce savoir contingent de situations toujours changeantes rend le métier d'éleveur risqué voire dangereux. Peu abordé entre les éleveurs euxmêmes, ce savoir est presque ignoré de la recherche et du conseil technique agricole comme si soigner et manipuler les bêtes étaient un allant de soi.

In a context of herds' enlargement, the breeder renews his relation to animals and his knowledge about animal management. Animal behavior is determined by usual practices and genetic heredity. The knowledge of the breeder lies in observing animal gregarious behavior, more expressed in extensive breeding. The breeder develops relations of cooperation with cows because it is not possible anymore to force them. He also has his tricks. This knowledge, contingent of always-changeable situations, makes the breeder job risky and dangerous. Modulated by few exchanges between the breeders themselves, he is almost unknown by agricultural research and advisory services as looking after animals is considered as going without saying.

\section{INDEX}

Keywords: knowledge, herd, docility, cattle handling, human/animal relationship, Saône-etLoire (France).

Mots-clés: savoir, troupeau, docilité, relation homme-animal, conduite des bovins, Saône-etLoire (France).

\section{AUTHOR}

\section{SANDRINE PETIT}

Ingénieure de recherche, géographe, CESAER, AgrosupDijon, Inra, Université de BourgogneFranche-Comté 